\title{
Discrete spectrum of Alfvén ion-ion hybrid waves
}

T. Intrator, M. Vukovic, A. Elfimov, P. H. Probert, and G. Winz

Department of Nuclear Engineering and Engineering Physics, University of Wisconsin, Madison, Wisconsin 53706-1687

(Received 16 October 1995; accepted 4 December 1995)

In the Phaedrus-T tokamak [R. Majeski et al., Phys Fluids B 5, 2506 (1993)], Alfvén waves are indirectly driven by a fast wave antenna array. Small fractions of minority ions can couple Alfvén and ion-ion hybrid waves and have a large effect on the wave numbers accessible for a given launched frequency. A discrete spectrum and toroidal damping for these modes has been identified by measuring dispersion properties at the edge. Landau damping is predicted to be large and spatially localized and to be responsible for the experimentally observed electron heating (T. Intrator et al., "Alfvén ion-ion hybrid wave heating in the Phaedrus-T tokamak," to appear in Phys. Plasmas) and current drive near the core of the tokamak plasmas. (C) 1996 American Institute of Physics. [S1070-664X(96)02603-6]

\section{INTRODUCTION}

For a magnetized plasma with gradients in density or magnetic field, there have been many theoretical predictions that Alfvén waves can have a resonance ${ }^{1}$ and heat the electrons at a spatially localized surface., ${ }^{2,3}$ Some examples include the solar corona, ${ }^{4}$ in a tokamak, ${ }^{5}$ and in auroral arcs. ${ }^{6}$ Electron heating and current drive in the Alfvén regime is indirectly accessible in a tokamak using ICRF (ion cyclotron range of frequencies) fast wave antenna heating systems that take advantage of mode conversion from antenna launched fast magnetosonic wave fields.

In the Phaedrus-T tokamak core electron heating ${ }^{7-9}$ and current drive $\mathrm{e}^{10,11}$ has been observed in the Alfvén regime below the hydrogen cyclotron frequency. The plasma was nominally pure $\mathrm{H}^{+}$with impurity minority species $\mathrm{C}^{\mathrm{VI}}$. This electron heating was shown to be caused by Alfvén ion-ion hybrid waves ${ }^{8}$ by comparing high-power heating shots with low-power frequency $\omega$ swept measurements of wave number $k_{z}$ and dispersion relations $\omega\left(k_{z}\right)$ at the plasma edge.

In this paper we are concerned with the further analysis of these data, which shows for the first time the existence of discrete eigenmode spectra for these hybrid modes. This is similar to the well-known discrete spectrum for Alfvén waves in nominally one species plasmas, as measured on many devices, including PRETEXT, ${ }^{12}$ Tokamak de Chauffage Alfvén TCA, ${ }^{13}$ Torus of the University of Sydney TORTUS, ${ }^{14}$ Tokamak Experiment for Technically Oriented Research (TEXTOR). ${ }^{15}$ The discrete Alfvén spectrum follows from many theoretical treatments of Alfvén waves, which include boundary conditions. ${ }^{16,17}$ A cylindrical model, not a toroidal one, appears to be adequate to explain the data shown in this paper, even though the Phaedrus-T tokamak has significant toroidicity at an aspect ratio $A \approx 3.7$.

A low-power frequency sweep experiment was used to measure the continuous and discrete dispersion properties, wave damping, and a relation between wave damping and fluctuations in the ensemble averages of measured wave numbers. This relation will be referred to later as a statistical fluctuation-dissipation relation. This wave behaves like an Alfvénic wave associated not with the nominally pure hydrogen ion species, but with an ion-ion hybrid Alfvén con- tinuum. The presence of impurity ions such as fully stripped $\mathrm{C}^{\mathrm{VI}}, \mathrm{D}^{+}$create a gap in the dispersion curve near the ion minority gyrofrequency and add another mode above the ion-ion hybrid frequency, as well as changing the discrete Alfvén spectrum. This approach is a different way of looking at the well-known ion-ion hybrid wave spectrum, ${ }^{8}$ and is not limited to the hydrogen majority plasmas that we used in Phaedrus-T.

\section{ALFVÉN AND ALFVÉN-ION-ION-HYBRID MODES}

When a plasma equilibrium is spatially inhomogeneous, the linearized equations of magnetohydrodynamics (MHD) allow waves with both continuous and discrete spectra. The second-order differential equations usually associated with the Alfvén waves in slab or cylindrical geometry admit two solutions. One solution has non-square-integrable spatial singularities ${ }^{18}$ located about equilibrium magnetic surfaces, and is associated with the continuum component. The term continuum refers here to the continuous range of frequencies for which the Alfvén resonance can exist in a plasma. Boundary conditions can be easily satisfied because the singular solutions are small everywhere, except at the resonance location. For this Alfvén-type resonance that we will be discussing, the dominant fields in cylindrical geometry are radial electric field $E_{r}$ and azimuthal magnetic field $B_{\theta}$ and

$$
E_{r} \propto B_{\theta} \propto\left(r-r_{0}\right)^{-1},
$$

where $n_{0}$ is the resonant radius. The other solution is regular (i.e., nonsingular), and if boundary conditions can be satisfied, these regular solutions lead to a discrete spectrum for the Alfvén waves. These solutions will form the discrete radial eigenmode spectrum that we discuss later for Alfvénic waves in Sec. IV.

For the cold plasma case $\left(m_{e} \rightarrow 0\right)$ and if the equilibrium plasma parameters are constant along magnetic field lines, the resonance condition manifests itself as a singularity in perpendicular wave number,

$$
N_{\perp}^{2}=\frac{\left(S-N_{\|}^{2}\right)^{2}-D^{2}}{S-N_{\|}^{2}},
$$

where 


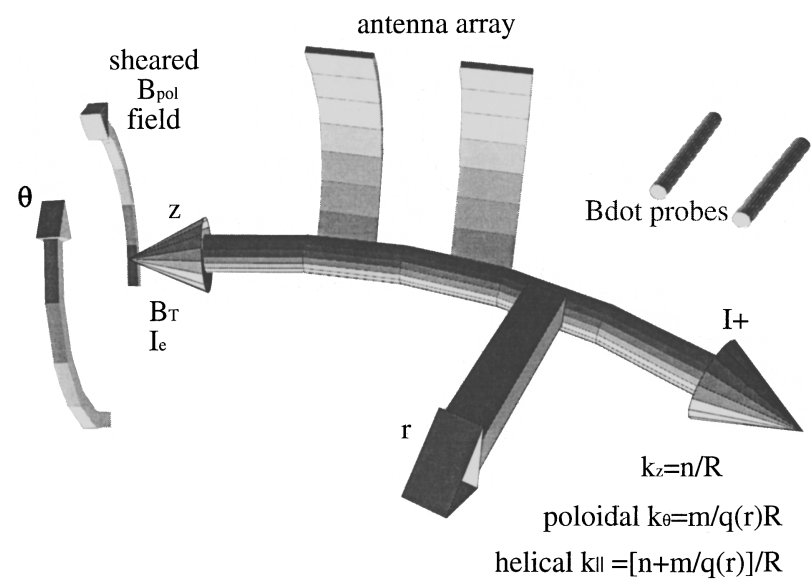

FIG. 1. Schematic of the basic cylindrical $(r, \theta, z)$ geometry, where $z$ is the toroidal direction parallel to the background magnetic field $B_{0}$ and the electron tokamak current. The positive current flows in the direction of waves traveling the short way around the torus from antenna to B-dot probes. The antenna array straps and B-dot probes are situated on the low-field side, and the sheared $B_{\theta}$ field is also indicated.

$$
S=1+\sum_{\sigma}\left[\omega_{p \sigma}^{2} /\left(\omega_{c \sigma}^{2}-\omega^{2}\right)\right]
$$

and

$$
D=\sum_{\sigma}\left[\left(\omega_{c \sigma} \omega_{p \sigma}^{2}\right) / \omega\left(\omega^{2}-\omega_{c \sigma}^{2}\right)\right]
$$

are the Stix parameters ${ }^{19}$ for the dielectric tensor. The perpendicular resonance including the sum over minority ion species contributions corresponds to $N_{\|}^{2}=S$, where the subscript $\sigma$ refers to all the electron and ion species. The perpendicular refractive index is $N_{\perp}=c k_{\perp} / \omega$, the parallel one is $N_{\|}=c k_{\|} / \omega, k$ is the wave number, $\omega$ is the wave angular frequency, $\omega_{p}$ is the plasma frequency, and $\omega_{c}$ is the cyclotron frequency.

Since the Alfvén wave has flux surface symmetry and follows helical magnetic field lines, our launched $k_{z}$ has a corresponding theoretical parallel wave number,

$$
k_{\|}=k_{z}+\frac{m}{q(r) R_{0}}=\frac{n+m / q(r)}{R_{0}},
$$

where $n$ and $m$ are, respectively, the toroidal and poloidal mode numbers, $R_{0}$ is the major radius on the axis where the minor radius $r=0$, and the safety factor is $q(r)$. A schematic of this cylinder geometry is shown in Fig. 1.

In the usual picture of the Alfvén resonance, the cylindrically symmetric density and $B_{\theta}$ gradients allow the local Alfvén speed, $v_{\mathrm{A}}(r)=B(r) /\left[\mu_{0} m_{i} n(r)\right]^{1 / 2}$ and frequency $\omega_{\mathrm{A}}(r)$ to match the pump field phase speed $v_{\emptyset z}$,

$$
\omega_{\mathrm{A}}^{2}(r)=\frac{[n+m / q(r)]^{2}}{R^{2}}\left(1-\frac{\omega^{2}}{\omega_{c i}^{2}}\right) v_{\mathrm{A}}^{2}(r),
$$

where the ion cyclotron frequency is $\omega_{c r}(r=0)$. If $v_{\emptyset z}$ $<v_{e}$, theory predicts ${ }^{16,20,21}$ mode conversion to a kinetic Alfvén wave (KAW), with substantial Landau damping onto the electrons.
In the Phaedrus-T high-power radio frequency (RF) heating experiment ${ }^{7}$ that we mention in the Introduction, the ion species mix consisted of a majority $\mathrm{H}$, with a heavier minority of impurity carbon that could not be reduced below $n_{\mathrm{C}} / n_{\mathrm{H}} \approx 1 \%-2 \%$. The heating and current drive effects were largest at $\omega \approx 0.7 \omega_{c \mathrm{H}}(r=0)$. This fact and others led to our suspicion that the observed electron heating was due to an Alfvénic ion-ion hybrid wave, and motivated a series of low RF power wave physics experiments. We added heavier ion species fractions such as deuterium, and covered the range $n_{\mathrm{D}} / n_{\mathrm{H}} \approx 0 \%-200 \%$. We believe that the mode converted wave is an Alfvén wave associated not with the nominally pure hydrogen ion species, but with a hybrid wave that follows from the coupling of the minority species with the Alfvén continuum. These Alfvén ion-ion hybrid waves occur at frequencies above the ion-ion hybrid frequency $\omega_{i i}$,

$$
\frac{\omega_{i i}^{2}}{\omega_{c 2}^{2}}=\frac{1+\rho_{z} / \Omega_{z}}{1+\rho_{z} \Omega_{z}},
$$

where the ion gyrofrequencies for species 1 and 2 are $\omega_{c 1}$ and $\omega_{c 2}, \rho_{z}=n_{z} m_{z} /\left(n_{\mathrm{H}} m_{\mathrm{H}}\right)$ and $\Omega_{z}=\omega_{c 2} / \omega_{c 1}=Z m_{\mathrm{H}} / m_{z}$ are, respectively, the mass density and gyrofrequency ratios for ion species 2 with respect to ion species 1 (species 1 is hydrogen here).

The theoretically expected dispersion for the pure $\mathrm{H}$ Alfvén continuum,

$$
\frac{\omega}{\omega_{c \mathrm{H}}}=\frac{\kappa_{\|}}{\left(1+\kappa_{\|}^{2}\right)^{1 / 2}},
$$

asymptotes to $\omega \approx \omega_{c \mathrm{H}}$ as $\kappa_{\|}$gets large. The toroidal and parallel wave numbers $k_{z}$ and $k_{\|}$are normalized to the ion skin depth at $r=0$ so that $\kappa_{z}=k_{z} c / \omega_{p \mathrm{H}}(r=0)$ and $\kappa_{\|}$ $=k_{\|} c / \omega_{p \mathrm{H}}(r=0)$, where $\omega_{p \mathrm{H}}$ is the hydrogen ion plasma frequency.

The presence of impurity ions such as C, D adds another mode to the Alfvén continuum dispersion ${ }^{22}$ above $\omega>\omega_{i i}$, and a gap at the ion gyrofrequency of the minority species $\omega_{c 2}<\omega<\omega_{i i}$, along with the Alfvén branch associated with the lower gyrofrequency ion, and changes the discrete Alfvén spectrum as well. ${ }^{23}$ For simplicity, we will refer to the lower branch as Alfvénic and the upper one as the hybrid branch. Borg and $\mathrm{Cross}^{24}$ may have observed these types of waves in the small tokamak TORTUS.

Elfimov ${ }^{23}$ et al. Used a cold plasma local approximation (i.e., a slab model) for the cylinder hybrid Alfvén continuum and showed that the $N_{\|}^{2}=S$ condition can be written down analytically as

$$
\begin{aligned}
& \left(\frac{\omega_{A \mathrm{H}}^{ \pm}}{\omega_{c \mathrm{H}}}\right)^{2} \\
& \quad=\frac{1+\rho_{z}+\xi \pm\left\{\left[1+\rho_{z}+\xi\right]^{2}-4 \kappa_{\|}^{2}\left[\rho_{z}+A^{2}\left(1+\kappa_{\|}^{2}\right)\right]\right\}^{1 / 2}}{2\left[\rho_{z}+A^{2}\left(1+\kappa_{\|}^{2}\right)\right]} .
\end{aligned}
$$

The left-hand side is the Alfvén hybrid frequency spectrum normalized to the hydrogen cyclotron frequency as a function of wave number where $A=\Omega_{z}^{-1}=m_{z} / Z m_{\mathrm{H}}, \quad \xi=\kappa_{\|}^{2}(1$ $+A^{2}$ ). The "extra" hybrid mode in Eq. (7) is the finite $k_{z}$ 
generalization of the well-known Buchsbaum-Bers ${ }^{25}$ ionion hybrid resonance. At $\omega=\omega_{i i}$ we have the parallel cutoff limit $k_{z} \rightarrow 0$ of the perpendicular resonance $k_{r} \rightarrow \infty$. Typically in the tokamak case, where the standard approximation is a slab model with gradients in $B(r)$ and $n(r)$ perpendicular to the background $B_{0}$ field, a local estimate with $k_{z}$ fixed and variable $k_{r}$ is used. For our dispersion relation approach in Eq. (7), we have fixed $k_{r}$ and solved for $k_{z}$. This is a different way of looking at an old problem.

In the following sections we will display two base cases for comparison, with nominally pure hydrogen $n_{\mathrm{C}} / n_{\mathrm{H}} \approx 1.4 \%$ during Ohmic discharges, compared with a different case including added minority $n_{\mathrm{D}} / n_{\mathrm{H}} \approx 25 \%$, which from Eq. (5) is equivalent to $n_{\mathrm{C}} / n_{\mathrm{H}} \approx 4.25 \%$.

\section{DESCRIPTION OF EXPERIMENT}

Phaedrus- $\mathrm{T}$ is a medium-sized tokamak ${ }^{26}$ with a major radius $R_{0}=0.93 \mathrm{~m}$, minor radius $a=0.25 \mathrm{~m}$, with a central electron temperature $T_{e}(0) \approx 400-600 \mathrm{eV}$. In order to investigate the wave physics, we performed low RF power experiments on standard Ohmic tokamak discharges, where RFinduced core electron heating was observed. ${ }^{7-9}$ The plasma current was $I_{p} \approx 70 \mathrm{kA}$, edge $q(a) \approx 3.7$, line average density $\left\langle n_{e}\right\rangle \approx 8 \times 10^{18} \mathrm{~m}^{-3}$. A two strap fast wave antenna array had two toroidally spaced poloidal current straps driving low phase velocity wave fields. The frequency was swept from far below to above $\omega_{c \mathrm{H}}$, while maintaining strap phasing of $b_{\text {ant }}=[0, \pi]$. The measured peak and minimum of the vacuum $k_{z}$ spectrum were $k_{z} \approx 0.2$ and $0.0 \mathrm{~cm}^{-1}$, respectively. This experiment was very similar to a frequency sweep experiment carried out on TEXTOR, ${ }^{13}$ where they identified a discrete MHD Alfvén spectrum.

Four $30 \mathrm{~ms}$ sweeps consisting of a $20 \mathrm{~ms}$ sweep plus 10 ms of dead time were carried out per shot. The 200 ms tokamak shot typically had a plasma flat top period of $100 \mathrm{~ms}$, so that at least several of the sweeps had usable data. The frequency tracking linearity of the sweep was verified by directly digitizing the frequency sweep time record. Typical frequency resolution was set by the digitizer sample rate of $40 \mathrm{~K}$ sample/s, which for a 2-10 MHz sweep over $20 \mathrm{~ms}$ corresponded to $10 \mathrm{kHz}$ change in sweep frequency during each sample. This may have limited our resolution of discrete eigenmode structures that appear in the data shown in this paper.

The frequency source was a Wavetek 178 frequency sweepable signal generator, power split to provide both a local oscillator reference for a broadband vector voltmeter and RF driver signal for a low-power $0^{\circ}-180^{\circ}$ hybrid splitter. The split signal drove two broadband power amplifiers, which excited the two antenna straps through matched lengths of cable with $[0, \pi]$ phasing. $^{7}$ The antennas were driven through a voltage measurement feedpoint, inserted so as to avoid the series matchbox capacitor, and drive the parallel combination of the strap inductance and parallel matching capacitor directly. The parallel capacitor was adjusted to the minimum value so that the resonance was at the highest possible frequency $(>25 \mathrm{MHz})$.

Amplitudes and phases of the antenna drive signals, antenna circulating currents, and B-dot probes ${ }^{23}$ in the plasma were monitored. Group delays $\tau_{G}=\partial \phi / \partial \omega$ derived from signal phase $\phi(\omega)$ corresponding to cable length and electronic delays were measured as a function of frequency, and were normalized out of the phase measurements. Circulating current was of the order of $4 A_{\mathrm{rms}}$.

Wave magnetic fields were measured with $\mathrm{B}-\mathrm{dot}^{27}$ probes at a minor radius of $r=22.5 \mathrm{~cm}, 2.5 \mathrm{~cm}$ on the plasma side of the limiter, i.e. in the plasma on the equatorial plane, approximately $90 \mathrm{~cm}$ toroidally from the antenna array, and $630 \mathrm{~cm}$ the other way around the torus.. Assuming that there were damped waves propagating as $B\left(\omega, k_{z} ; z\right)$ $\approx B e^{i\left[k_{z}(\omega) z+m \theta-\omega t\right]}=B e^{i \phi(\omega)}$, we inferred a complex-valued $k_{z}(\omega)=\operatorname{Re}\left(k_{z}\right)+i \cdot \operatorname{Im}\left(k_{z}\right)$ from the signal phase and amplitudes ensemble averaged over many shots. We inferred the wave number with a two probe $k_{z}(\omega)$ measurement, where $k_{z}$ was assumed to be the phase difference divided by the probe separation along a toroidal arc. We note that the phase speed $v_{b z}=\omega / k_{z}$ as well as the group speed $\vec{v}_{G}=\partial \omega / \partial \vec{k}$ can be inferred from frequency swept data. In this case, the direction from the antenna to the B-dot probes corresponded to $k_{z}<0$, where $k_{z}>0$ would be parallel to the background $B_{0}$ field and antiparallel to the plasma (ion) current, as seen in Fig. 1.

From the phase shifts of the shear field component $B_{\theta}$, we measured the average values of the wave dispersion properties $k_{z}(\omega)$. Statistical properties of the collective modes, wave dispersion, and damping properties were accessible by ensemble averaging the realizations over many tokamak shots, and multiple sweeps within shots, and over the neighborhood frequency points to each composite dataset.

\section{FREQUENCY SWEEP DATA}

\section{A. Dispersion}

Even though the fast wave antenna is nominally a compressional $B_{z}$ launcher, substantial shear fields are launched ${ }^{28}$ from feeds and near fields, and the shear Alfvén wave (SAW) we seek has principally shear polarization. It is well known that near an Alfvén resonance zone, the wave group speed slows down and the $B_{\theta}$ wave energy "piles up" and swells. Historically the experimental signature of Alfvén waves, even at the core of a largely inaccessible plasma, has been observed to be the edge $B_{\theta}$ wave field, ${ }^{12-14,29}$ so our use of edge B-dot probes to measure core wave properties has some tradition behind it.

For a nominally pure $\mathrm{H}^{+}$plasma with $\phi_{\text {ant }}=[0, \pi]$, the results of an ensemble-averaged $k_{z}(\omega)$ measurements are shown in Fig. 2, where toroidal mode number $n=k_{z} R_{0}<0$ is indicated on the top axis and normalized wave number $\kappa_{z}$ $=k_{z} c / \omega_{p \mathrm{H}}(r=0)$ on the bottom. Frequency is normalized to the ion gyrofrequency on axis $\omega_{c \mathrm{H}}(r=0)$. The theoretically expected dispersion for the pure $\mathrm{H}$ Alfvén continuum is indicated with a dotted line. We plot the relation given by the slab model equation (6) for $\omega\left(\kappa_{\|}\right) / \omega_{c \mathrm{H}}$ as a function of $\kappa_{z}$, for $m=-1$, where we have used Eq. (3) and invoked an average value of the safety factor $\langle q\rangle=2$. We have plotted the $m=-1$ dispersion, because this is what is expected for the mode converted shear wave, even though the directly launched compressional $B_{z}$ wave fields have $m=+1$. Both 


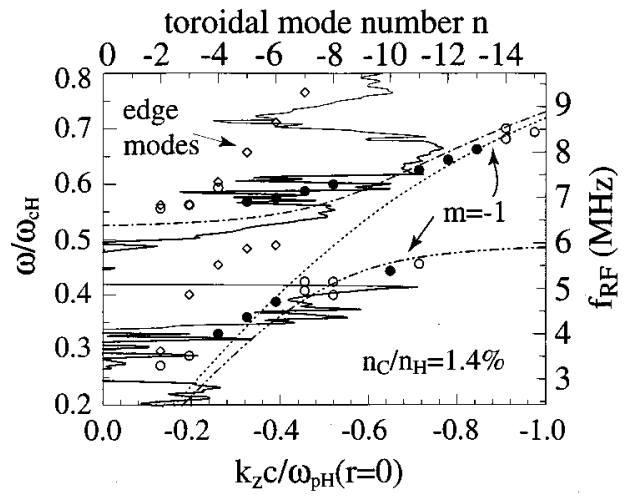

FIG. 2. Axial wave number $k_{z}$ and toroidal mode number for the low-power sweep experiment with $B_{0}=0.8 \mathrm{~T},\left\langle n_{e}\right\rangle=8 \times 10^{18} \mathrm{~m}^{-3}$, estimated $n_{\mathrm{C}} / n_{\mathrm{H}}=1.4 \%$. Here $k_{z}$ is inferred from B-dot $B_{\theta}$ data, and is shown as a dispersion plot of normalized frequency $\omega / \omega_{c \mathrm{H}}$ versus the normalized wave number $\kappa_{z}=k_{z} c / \omega_{p \mathrm{H}}(r=0)$. The calculated slab model continuum boundaries for $m=-1$ are shown for the pure hydrogen Alfvén mode (dotted line), the Alfvénic branch (dash-dotted) below $\omega / \omega_{c \mathrm{H}}=\omega / \omega_{c \mathrm{D}} 0.5$ and hybrid branch (dash-dot-dotted) above $\omega_{i i}$ for $\omega / \omega_{c \mathrm{H}}>0.53$. The ISMENE calculated discrete modes are shown in circles where the filled circles correspond to the largest calculated loading. The ISMENE calculated surface eigenmodes for both Alfvénic and ion-ion hybrid branches corresponding to surface quasielectrostatic waves (SQEW) are also indicated with diamonds. The B-dot data do not resemble the SQEW predictions.

are within the broad measured poloidal mode spectrum of $-2<m<2$ that our antenna launches.

The split hybrid Alfvén continua from the slab model equation (7), including our actual impurity fraction of $1.4 \%$ carbon, has been calculated for the perpendicular resonance $N_{\|}^{2}=S$. The two split branches include the hybrid branch above $\omega_{i i}$ (dot-dashed) and the Alfvénic branch below $\omega_{c \mathrm{D}}$ $=\omega_{c \mathrm{H}} / 2$ (dash-dot-dotted), as shown in Fig. 2, along with the frequency gap in between. The hybrid branch asymptotes to $\omega=\omega_{i i}$ at small $\left|k_{\|}\right|=\left|k_{z}+m / q R_{0}\right| \rightarrow 0$, which for $m=-1$ corresponds to $k_{z}=-m / q R_{0} \approx$ or $\kappa_{z} \approx 0.03>0$, i.e., to the left of the vertical axis at $\kappa_{z}=0$. The Alfvénic branch (dash-dotdotted) on the bottom half of Fig. 2 asymptotes to $\omega$ $=0.5 \omega_{c \mathrm{H}}$ at large $\left|\kappa_{\|}\right|$, i.e., as $\kappa_{z} \rightarrow-1$. For these discussions we will refer to the continuum and its boundary as the Alfvén continuum, even though the reader must bear in mind that we usually will be referring to the ion-ion hybrid branch of the split Alfvénic spectrum.

The hybrid (dot-dashed) and Alfvénic (dash-dotdotted) lines are the slab model Alfvénic continua evaluated for the on axis $B_{0}$ and density. This also approximately corresponds to the lower-frequency edge of the continua in a cold plasma MHD cylinder model specified by the highest density on axis at $r=0$. A kinetic calculation of antenna loading including $q(r)$ profiles could also be displayed as a contour on a dispersion diagram like Fig. 2. This continuum edge turns out to be more consistent with discrete modes, which are described in the next section. We note in passing that there are also backward propagating waves in the "forbidden" frequency gap $\omega_{c \mathrm{D}}<\omega<\omega_{i i}$, which turn out to be highly damped in the toroidal direction.

In Fig. 3(a) we show the phase data from the B-dot probes along with calculations, but for an expanded scale in the region of interest. The measured dispersion fits approxi-

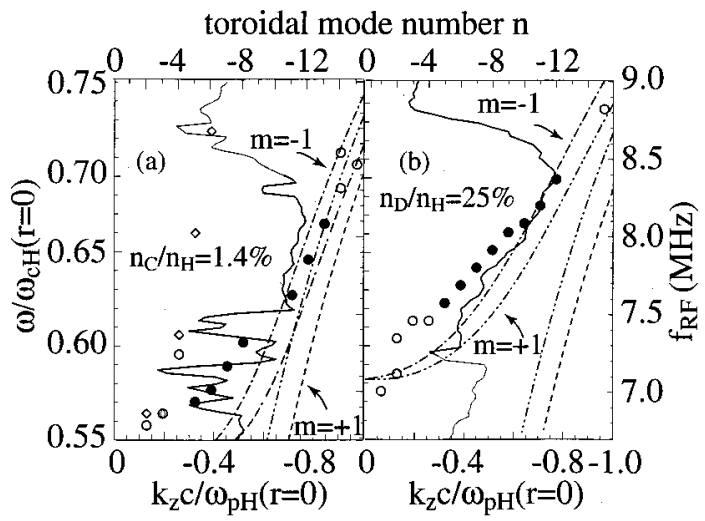

FIG. 3. Dispersion plots are shown with expanded frequency scale to show the hybrid branch in more detail. Circles indicate ISMENE calculated discrete modes, filled circles indicate modes with the largest predicted loading, and diamonds correspond to the calculated SQEW edge low-density modes. Normalized frequency is $\omega / \omega_{c \mathrm{H}}(r=0)$ on the hybrid branch that is above $\omega_{i i}$ and is plotted against normalized wave number $\kappa_{z}=k_{z} c / \omega_{p \mathrm{H}}$ for the low-power sweep experiment with $B_{0}=0.8 \mathrm{~T},\left\langle n_{e}\right\rangle=8 \times 10^{18} \mathrm{~m}^{-3}$. Actual sweep frequency in $\mathrm{MHz}$ is on the right-hand axis. The poloidal mode numbers used for the slab model calculated continuum edge are keyed as $m=-1$ hybrid mode (dash-dotted), $m=+1$ hybrid mode (dash-dashdotted), $m=-1$ Alfvénic mode (dash-dot-dotted), and $m=+1$ Alfvénic mode (dash-dashed). (a) Estimated minority species density fraction $n_{\mathrm{C}} / n_{\mathrm{H}}=1.4 \%$. (b) Estimated $n_{\mathrm{D}} / n_{\mathrm{H}}=25 \%$.

mately in the region for the slab fluid model of Eq. (7). The range in slab model $\omega\left(\kappa_{z}\right)$ for the ion-ion hybrid branch corresponding to the $m=-1$ (dash-dotted) and $m=+1$ (dash-dash-dotted) is shown in Fig. 3(a). Similarly, the Alfvénic branch corresponding for $m=-1$ (dash-dotdotted) and $m=+1$ (dash-dashed) is also shown in Fig. 3(a). The circles and diamonds correspond to the predicted discrete eigenmode mode structure that is seen more clearly in the measured damping and discussed in the next section.

Figure 3(b) shows the same type of dataset as Fig. 3(a) for a dispersion curve displayed as $\omega / \omega_{c \mathrm{H}}(r=0)$ vs $k_{z} c / \omega_{p \mathrm{H}}(r=0)$, but for a substantial fraction of D impurity, $n_{\mathrm{D}} / n_{\mathrm{H}}=25 \%$. The vertical scale has been expanded to show the region of interest and dispersion of the hybrid branch. The whole dispersion curve has moved upward in frequency with the gap now below $\omega \approx 0.6 \omega_{c \mathrm{H}}(r=0)$, as expected. We use the same labeling convention as for Fig. 3(a), and indicate slab model estimations for $m= \pm 1$ hybrid and $m= \pm 1$ Alfvénic branches. We will return to these figures when we discuss the discrete mode spectrum in the next section.

\section{B. Discrete mode structure}

The discrete spectrum of the Alfvénic and hybrid branches has been calculated with ISMENE. ${ }^{16,30}$ ISMENE is a one-dimensional (radial) cylinder model computer code that includes kinetic effects and radial derivatives equivalent to second order in $k_{r}^{2} \rho_{l}^{2}$, axial plasma current, and mode conversion and Alfvén continuum, kinetic Alfvén waves (KAW), ion Bernstein waves (IBW), and discrete Alfvén eigenmodes (DAE). On a dispersion diagram $\omega(k)$ such as Figs. 2 and 3, the calculated discrete modes form a series that crosses the $m=-1$ continuum boundary. In a slab or MHD cylinder model, the Alfvén resonances can exist for values of $\omega, k$ 


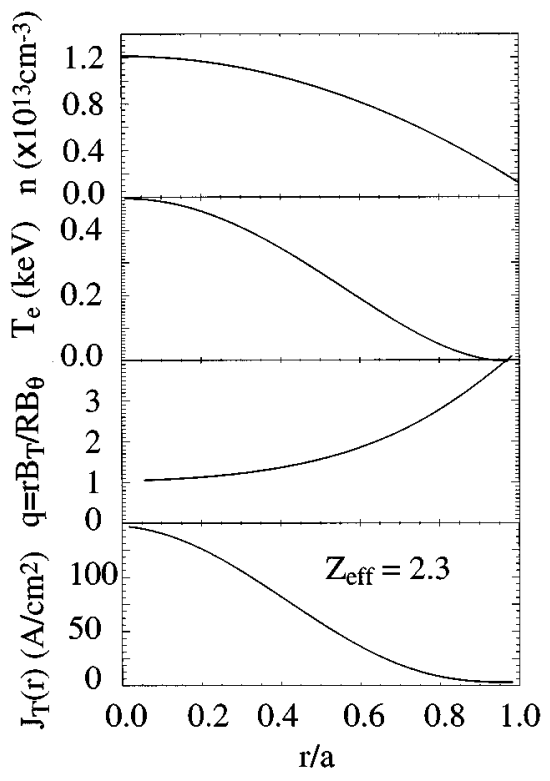

FIG. 4. For the ISMENE calculations we assumed radial profiles of density $n$, electron temperature $T_{e}$, safety factor $q$, and current density $J_{T}$, and $Z_{\text {eff }}=2.3$.

with phase speeds $\omega / k_{\|}$that can be no slower than those at the continuum boundary. On the $\omega-k$ dispersion diagram the Alfvén continuum, i.e., the spectrum of resonances lies above the $m=-1$ ion-ion hybrid continuum boundary, as shown by the dash-dotted lines in Figs. 2 and 3. The calculated discrete modes are not resonances, but eigenmodes that transition from surface wave DAE's at low $|n|$ to global Alfvén eigenmodes (GAE) at larger $|n|$. These ISMENE calculations were carried out as frequency scans for each assumed discrete $n$, and the frequency at peak loading was identified as the mode of interest. The radial profiles we assumed for the ISMENE calculations are shown in Fig. 4, where we use the dimensionless radius $\rho=r / a$. The density was taken to be parabolic $n_{e}(r) / n_{e}(0)=1-\rho^{2}$, electron temperature $T_{e}(r) / T_{e}(0)=\left(1-\rho^{2}\right)^{2}$, the ion temperature $T_{i}(r)$ was taken to be equal to $T_{e}(r)$, the loop voltage was taken to be $2 \mathrm{~V}$, and the effective ionic charge was $Z_{\text {eff }}=2.3$. The calculated Spitzer resistivity yields a current density $J(\rho)$ and safety factor $q(\rho)$ profile that are consistent with our measured edge $q(\rho=1)$ and measured $T_{e}(\rho)$ for the Phaedrus-T tokamak.

In Fig. 3 the calculated discrete spectrum is indicated by the circles, where the filled ones correspond to the modes with the largest predicted loading. The discrete modes lie near the slab approximation $\left(B_{0}, n\right.$ at $\left.r=0\right)$ continuum edge for both Alfvén and hybrid branches. The continuum, as explained in Sec. II, is the continuous frequency range that admits Alfvén resonant solutions inside the bounded plasma cylinder. The lower-frequency bound of the Alfvén continuum corresponds to the maximum density at the core, which in turn gives the lowest Alfvén speed and lowest Alfvén frequency according to Eq. (4). In Fig. 2 the Alfvén branch for the swept frequency data in Fig. $2\left(\omega \ll \omega_{i i}, \omega\right.$ $<\omega_{c \mathrm{D}}$ portion) probably includes discrete modes associated

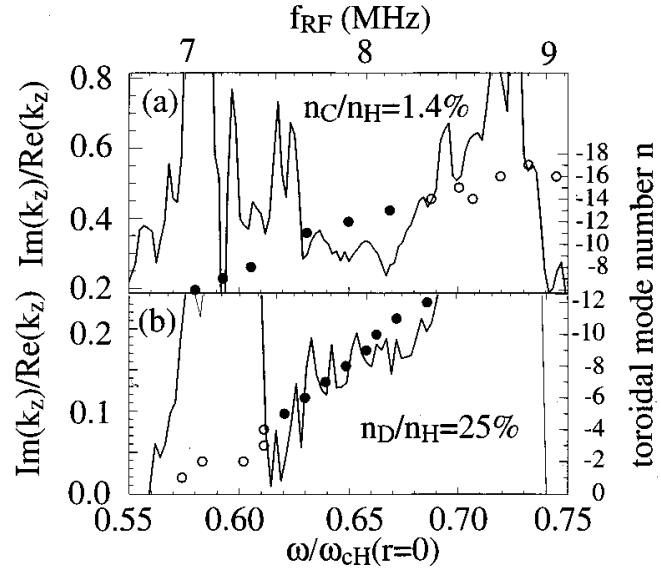

FIG. 5. Fractional damping given by the measured ratio $\operatorname{Im}\left(k_{z}\right) / \operatorname{Re}\left(k_{z}\right)$ for frequency swept data, where we show the hybrid branch on an expanded frequency scale. The circles indicate ISMENE calculated discrete modes, with filled circles corresponding to those modes with the largest predicted loading. The right-hand axis shows the discrete toroidal mode number $n$, which also corresponds to the data in Figs. 3(a) and 3(b). (a) For estimated $n_{\mathrm{C}} / n_{\mathrm{H}}=1.4 \%$, there is a correspondence between ISMENE calculated discrete modes and local minima in damping for toroidal modes $n=-7$ to -8 and $n=-11$ to -16 . (b) For estimated $n_{\mathrm{D}} / n_{\mathrm{H}}=25 \%$, there is a correspondence between ISMENE calculated discrete modes and local minima in damping for toroidal modes $n=-4$ to -12 .

with partially stripped ion species, but still qualitatively looks like an Alfvén dispersion characteristic.

Since there are $n(r)$ and $q(r)$ gradients in the cylinder, the dispersion from Eq. (7) only indicates the lower edge of the highest-density $\omega_{\mathrm{A}}(r=0)$ [see Eq. (4)] in the Alfvén continuum. But discrete surface modes associated with the lowdensity plasma edge gradient can also be calculated with ISMENE and are indicated with diamonds in Figs. 2 and 3. The swept frequency data coincide with the dispersion relation associated with the lower Alfvén continuum edge and high-density core, and not the calculated discrete surface modes associated with the low-density edge (sometimes called surface quasielectrostatic modes $\left.{ }^{13,16}\right)$.

In Fig. 5(a) we show the damping $\operatorname{Im}\left(k_{z}\right) / \operatorname{Re}\left(k_{z}\right)$ measured with pairs of probes on an expanded scale, for a nominally pure $\mathrm{H}$ plasma. Calculated DAE modes occur at $n-6$ to -8 and $n=-11$ to -16 on the hybrid branch, and they correlate with measured dips in damping. The sharpness of the discrete structure may be blurred from the spread $( \pm 4 \%)$ in density for the shots and multiple frequency sweeps in this data ensemble, and possibly toroidicity effects.

In Fig. 5(b) we show the frequency spectrum of damping $\operatorname{Im}\left(k_{z}\right) / \operatorname{Re}\left(k_{z}\right)$ for the case of substantial D impurity fraction, where $n_{\mathrm{D}} / n_{\mathrm{H}} \approx 25 \%$. The circles represent the discrete modes predicted by ISMENE, with solid ones having the largest predicted loading. The calculated DAE $(n=-5$ to -12$)$ discrete modes on the hybrid branch $d o$ correlate with dips in fractional damping, as expected for resonant modes.

\section{Fluctuation dissipation relation}

For many-particle systems in or near thermodynamic equilibrium, the fluctuation-dissipation theorem furnishes a connection between the dissipation of excited modes and the 
correlation function of the forces or quantities that drive that dissipation. This theorem is a fundamental property of many statistical systems and connects the equilibrium properties (fluctuations) with irreversible processes (dissipation) that drive the system away from equilibrium. In our case the wave number $k_{z}$ measurements follow from a two-point correlation functions that have been ensemble averaged, and Figs. 2 and 3 show our measured frequency spectra of $k_{z}$. The dissipation follows from the imaginary part of the linear response function, or the measured wave damping frequency spectrum, such as shown in Fig. 5. Landau damping is presumed to couple wave energy to the electrons, which results in the electron heating we measure. ${ }^{7-9}$ Therefore wave energy and momentum is carried away from the waves via Coulomb electron-electron collisions.

We make two assumptions about the statistics. First, that the measurement time is much shorter than the dissipation time, so that it is the dissipation that dominates the decorrelation process. The correlation measurement time scale is presumed to be of the order of the transit time of the Alfvén wave between the two B-dot probes, or approximately $\tau_{\text {corr }} \approx 50 \mathrm{~ns}$. The dissipation time for electron-electron collisions from Spitzer resistivity is approximately $\tau_{\text {diss }} \approx 5-20$ $\mu \mathrm{s}$. Second, we assume that the process is stationary, i.e. that the correlation depends only the time (or spatial) separation of the measurements. Thus, successive frequency sweeps every $30 \mathrm{~ms}$ are uncorrelated and separated by a time longer than the dissipation time. The sample time per data point during each sweep was $25 \mu \mathrm{s}$, which was of the order of the dissipation time, so that there is some utility in regarding data from successive sweeps as equivalent to a time history of correlation measurements.

Here $\operatorname{Re}\left(k_{z}\right)$ was calculated as an average over an ensemble of datasets. For the many realizations in the ensemble, the distribution of calculated wave numbers also had some spread about the mean. We will express this jitter in the data as a standard deviation $\sigma_{\kappa}(t)$ time record,

$$
\sigma_{k}(t)=\left(\langle\kappa(t)\rangle^{2}-\left\langle\kappa^{2}(t)\right\rangle\right)^{1 / 2},
$$

in the same units as $\kappa$, where the brackets \langle\rangle signify an ensemble average.

In Fig. 6(a) we compare the measured damping fraction $\operatorname{Im}\left(k_{z}\right) / \operatorname{Re}\left(k_{z}\right)$ (dashed lines) from Fig. 5 and the calculated standard deviation $\sigma_{\kappa}$ (solid lines), both as a function of the swept frequency. Increased damping correlates well with increased jitter $\sigma_{\kappa}$ in the average wave number for this nominally pure $\mathrm{H}$ plasma. The indicated discrete modes also correspond to local $\left(\omega, k_{z}\right)$ regions with reduced damping and $\sigma_{\kappa}$. The standard deviations $\sigma_{\kappa}$ of the calculated $\operatorname{Re}\left(k_{z}\right)$ from the ensemble of $n_{\mathrm{D}} / n_{\mathrm{H}}=25 \%$ datasets is shown in units of $\kappa$ in Fig. 6(b). The $\sigma_{k}$ jitter spectrum shown in Fig. 6(b) increases when wave damping increases, and also where the dispersion relation transitions to anomalous dispersion character. By anomalous we mean that the group velocity appears to be unphysically large or negative, as is apparent from inspection of Figs. 2 and 3. With large damping $v_{G}$ is not an appropriate description of a wave packet. ${ }^{31}$

These data indicate that some sort of fluctuation dissipation relation holds for the statistical ensemble of these

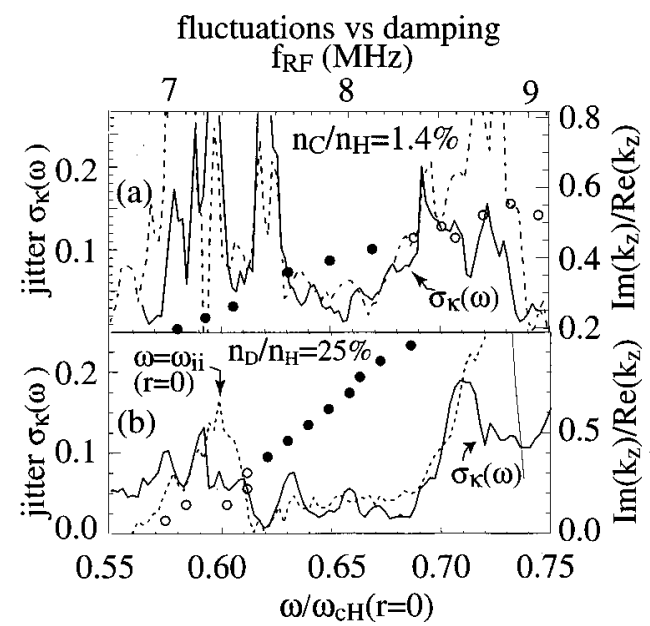

FIG. 6. Jitter frequency spectrum $\sigma_{\kappa}(\omega)$ is shown by the solid line in units given by the left-hand axis evaluated over the ensemble of the datasets. This is compared with fractional damping (dashed line) given by the measured ratio $\operatorname{Im}\left(k_{z}\right) / \operatorname{Re}\left(k_{z}\right)$ in units specified by the right-hand axis. The discrete mode numbers are the same as Figs. 3 and 5. (a) Estimated minority species density fraction $n_{\mathrm{C}} / n_{\mathrm{H}}=1.4 \%$. (b) For estimated $n_{\mathrm{D}} / n_{\mathrm{H}}=25 \%$.

damped waves. This also suggests that the dissipation in question is a truly irreversible departure from the equilibrium fluctuation spectrum, consistent with the electron heating observed $^{7-9}$ in Phaedrus-T at the same $\omega / \omega_{c \mathrm{H}}(r=0) \approx 0.7$. As will be shown in Sec. V, there is a connection between $B_{\theta}$ we measure at the edge, $E_{z}$ at the resonance location, and the associated electrostatic damping. Hence, there is a legitimate link between the $k_{z}$ correlation functions we invoke and the damping they engender. If some phase mixing phenomena or three-wave coupling was cascading the energy away from the region of interest, we would expect to see the "missing" energy correlated at some other frequency. This issue merits further investigation.

\section{Polarization and mode structure}

The ISMENE model we rely on for interpreting much of our data predicts wave polarizations that are qualitatively similar to our measurements. In this section we show probe data for the two cases of minority concentration and compare them with ISMENE results. For a nominally pure $\mathrm{H}$ plasma, Fig. 7 shows the magnitude of the $B_{\theta}$ data from a B-dot probe $90 \mathrm{~cm}$ (the "short" way around the torus) from the antenna. There is enhanced shear $B_{\theta}$ for the hybrid branch above $\omega>\omega_{i i}(r=0)$ and Alfvén branch below $\omega<\omega_{c \mathrm{D}}(r$ $=0)$. The discrete mode numbers predicted by ISMENE for $n_{\mathrm{C}} / n_{\mathrm{H}}=1.4 \%$ are indicated by the circles, filled ones having the largest predicted loading, with the $n<0$ values shown on the right-hand axis. Enhancement of $B_{\theta}$ is apparent for $n=-5,-6$ on the Alfvén branch and $B_{\theta}, B_{+}$for $n=-7,-8$, $-11,-12$ on the hybrid branch. As will be seen later, the higher-frequency region of the hybrid branch has more $B_{+}$ character, where $B_{+}=\left(B_{r}+i B_{\theta}\right) / \sqrt{2}$ is the left-hand circularly polarized (LHCP) component of the field. There are also waves (different polarization, $m$ ) in the frequency gap 


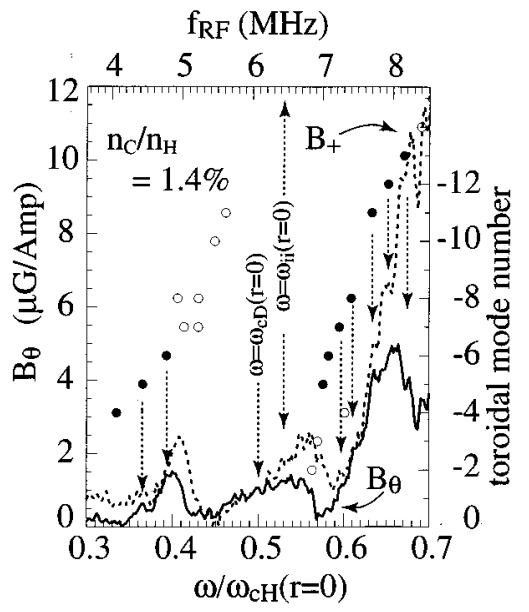

FIG. 7. Amplitudes of wave shear $B_{\theta}$ (solid line) and LHCP $B_{+}$(dashed line) fields from the frequency swept data of Fig. 6 for estimated $n_{\mathrm{C}} / n_{\mathrm{H}}=1.4 \%$, where the top axis indicates the frequency in $\mathrm{MHz}$. The circles indicate ISMENE calculated discrete modes, with filled circles corresponding to those modes with the largest predicted loading. Toroidal mode numbers appear on the right-hand axis. The dashed lines call attention to a correspondence between calculated discrete modes and local maxima in field amplitudes for $-n=5,6,7,8,11,12$, and 13 on both branches.

$\omega_{c \mathrm{D}}<\omega<\omega_{i i}$ apparently propagating "backward" $\left(k_{z}>0\right.$ from Fig. 2) the "long" way around the torus, with a pathlength of at least $650 \mathrm{~cm}$.

Figure 8 shows the wave polarization measured at the B-dot probe $90 \mathrm{~cm}$ from the antenna for $n_{\mathrm{D}} / n_{\mathrm{H}}=25 \%$ datasets. The shear $B_{\theta}$ (solid) and LHCP $B_{+}$(dashed) components are indicated. A change occurs in wave character from shear polarization in the small $k_{z}$, lower-frequency portion of the DAE series $(n \approx-7$ to -12$)$ to LHCP polarization for $\omega>\omega_{i i}$ GAE modes at $n=-15$. The global GAE mode is on the other side of the continuum edge from the DAE modes. The entire discrete series approaches the Alfvén continuum edge (see dispersion diagrams in Figs. 2 and 3), and after they cross the edge they become GAE's.

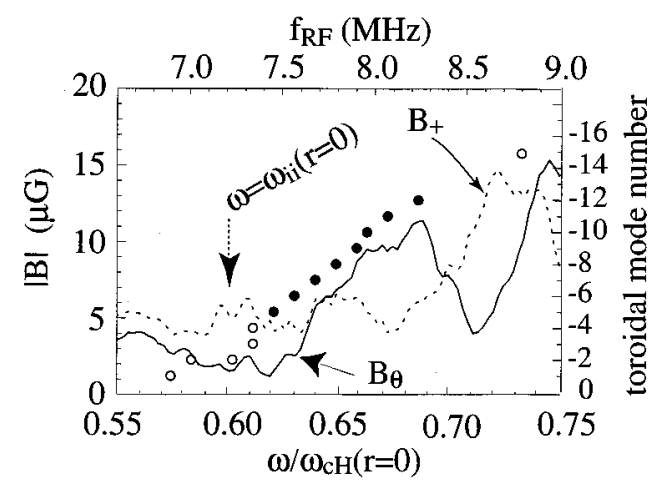

FIG. 8. Amplitudes of wave shear $B_{\theta}$ (solid line) and LHCP $B_{+}$(dashed line) fields from frequency swept data for estimated $n_{\mathrm{D}} / n_{\mathrm{H}}=25 \%$, where the top axis indicates the frequency in $\mathrm{MHz}$ and toroidal mode numbers are shown on the right-hand axis. The wave fields transition from shear to LHCP polarization as the frequency and wave number increase up the dispersion curve from 7.5 to $8.5 \mathrm{MHz}$, corresponding to Fig. 10. The ion-ion hybrid frequency $\omega_{i i}(r=0)$ is shown as well.

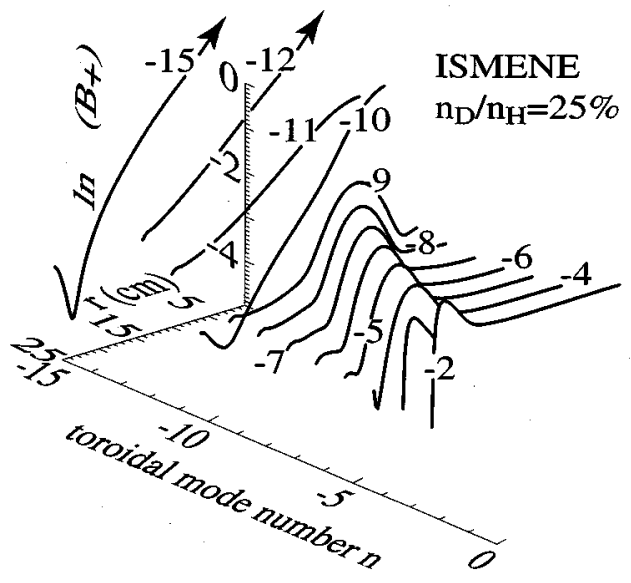

FIG. 9. Radial profiles of ISMENE calculated $B_{+}$fields are shown for each eigenmode with $n_{\mathrm{D}} / n_{\mathrm{H}}=25 \%$ equivalent to $n_{\mathrm{C}} n_{\mathrm{H}}=4.25 \%$. There is a transition between the discrete surface mode DAE character for low $|n|$ and core global eigenmode GAE character for larger $|n|$. Note the logarithmic scale. LHCP $B_{+}$field profiles are shown here, where scales for peak values of $B_{+}$ for $n=-12,-15$ have been suppressed to avoid compressing the vertical scale. For $|n| \geqslant 12$ the GAE's are characterized by LHCP polarization both in the core and at the edge.

The data in Fig. 8 can be compared with ISMENE calculations of the radial field profiles expected for these discrete modes. Figure 9 shows ISMENE calculated discrete Alfvén mode fields on the hybrid branch $\omega>\omega_{i i}$ for the LHCP field $B_{+}$as a function of discrete toroidal mode number $n$ and minor radius $r$. The plasma parameters were $T_{e}(0)=500 \mathrm{eV}$, $n_{\mathrm{D}} / n_{\mathrm{H}}=25 \%, m=-1$, the rest of the profiles as given in Fig. 4. Each $n$ corresponds to a different $\omega$ at the loading peak as we track a dispersion relation such as the one in Fig. 3. The GAE's are predicted to be LHCP near the core for $n \approx-8$ to -16 , as well as exhibit some surface wave LHCP fields at the edge. Figure 9 shows that as $|n|$ increases, edge $B_{+}$also increases gradually, and then as $n$ passes by $-10, B_{+}$ doubles. This is similar to the Fig. 8 data. To avoid compressing the vertical scale, peak values of $B_{+}$for $n=-12$, -15 are truncated at $r<7 \mathrm{~cm}$, so that $B_{+}$is considerably larger than it appears. The edge behavior is qualitatively similar to the Fig. 8 data.

Figure 10 shows ISMENE calculated $B_{\theta}$ radial profiles for DAE's. Inside the continuum the $B_{\theta}$ fields have more of a surface wave character and are dominantly shear polarized for $n \approx 0$ to -7 . The edge $B_{\theta}$ field comes up and then beyond $n=-7$ decreases, and then by $n=-11$ the $B_{\theta}$ crashes down again. This is the same trend, as Fig. 7 shows for edge $B_{\theta}$ fields during a frequency sweep, where $\omega$ or $|n|$ increase, although the match is not impressive. There is a transition between the surface wave character for low $\left|k_{z}\right|$ and $|n|$ $\approx 5-9$ to global wave character at increased $\omega$ and $|n| \approx 10-$ 12. The transitions in polarization in Fig. 7 and from the surface to global wave in Figs. 8 and 9 are apparent and occur for the minimally damped frequency range 7.5-8.5 MHz. In general, the DAE's have more shear $B_{\theta}$ and the GAE's have more LHCP $B_{+}$. 


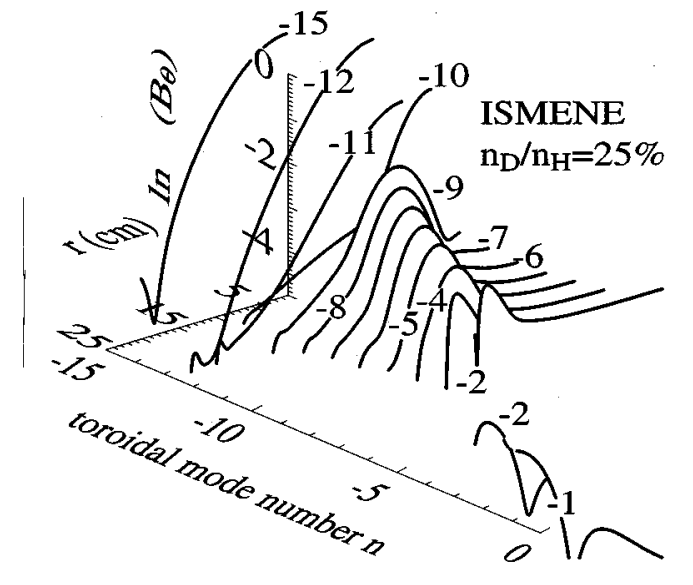

FIG. 10. Radial profiles of ISMENE calculated $B_{\theta}$ fields are shown for each eigenmode with $n_{\mathrm{D}} / n_{\mathrm{H}}=25 \%$. The DAE's tend to have more shear polarization at the edge and transition to shear fields that appear progressively farther into the core as $|n|$ increases.

\section{EDGE MEASUREMENTS OF CORE WAVE PHYSICS?}

It is striking that the dispersion characteristics measured on the edge of a tokamak plasma, as shown in Figs. 2 and 3, could track the wave physics in the core. We claim that the phase of the shear $B_{\theta}$ field appears to be a robust diagnostic of the core wave character. In Fig. 11, we show ISMENE ${ }^{16,30}$ calculations of the $B_{\theta}(r)$ phase as a function of radius at the maximum computed antenna loading, for example, eigenmodes at $n=-5,-10, m=-1$. These two cases show mode conversion near the edge $(n=-5)$ and the core $(n=-10)$. All these Alfvén and Alfvén-hybrid modes are variations of surface modes, with mode conversion from fast magnetosonic waves to KAW or IBW with significant $E_{z}$ and large $k_{r}$ and damping somewhere in the density gradient. Whether this kinetic ion-ion hybrid wave is in fact a KAW or IBW, or whether there exists a meaningful distinction at $N_{\|}^{2}=S$, is still an open question, and merits further investigation. The KAW and IBW both appear to be highly electrostatic, mostly perpendicular propagating waves with large $k_{r}^{2} \rho_{i}^{2}$.

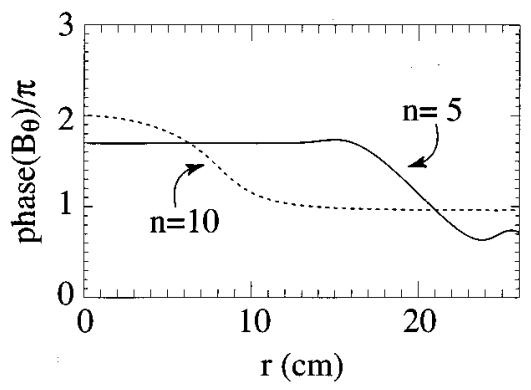

FIG. 11. Typical ISMENE calculations, showing radial profiles of the wave phase for shear $B_{\theta}(r)$ with $n_{\mathrm{D}} / n_{\mathrm{H}}=25 \%$. We show examples for discrete toroidal modes with fields in the core $(n=-10)$ and fields nearer to the edge $(n=-5)$. The jump in phase of $\pi$ radians at the Alfvén resonance location is evident, although it is not clear whether this should be called an IBW or KAW.

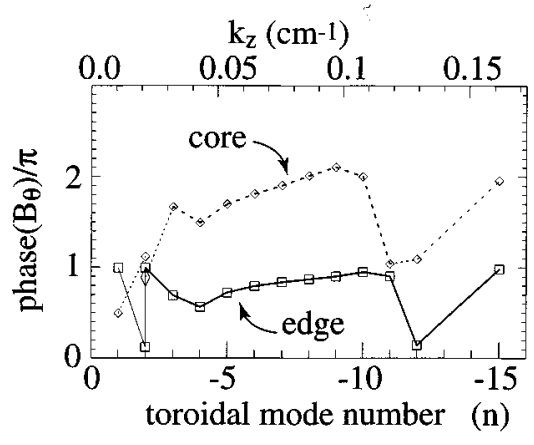

FIG. 12. ISMENE calculations of wave phase for each discrete toroidal eigenmode for $B_{\theta}$ evaluated at the edge (squares) and in the core (diamonds). The difference between the edge and core phase is a constant $\pi$ radians, except for $n=-2,-11$. Given the discrete eigenspectrum, an edge phase measurement of $B_{\theta}$ appears to be a useful diagnostic of core fields.

After launch from the antenna, the evanescent fast magnetosonic wave has a stationary phase at the edge. Near the resonance radius the wave $B_{\theta}$ field in the plasma acquires large local $k_{r}$ and during mode conversion phase shifts by $\pi$ radians across the resonance. Inside the resonance there is a stationary phase radial standing wave in the core. The $B_{\theta}$ field reversal arises at the KAW resonance zone analogous to the driven harmonic oscillator when the pump frequency crosses the resonant frequency. The $B_{r}$ field is continuous and slowly varying across this region, so that we can invoke Ampère's law in the region of $B_{\theta}$ discontinuity in the poloidal $r$ - $\theta$ plane,

$$
\int \boldsymbol{\nabla} \times \mathbf{B} \cdot d \mathbf{S} \approx \oint B_{\theta} \cdot d l \approx \int \mu_{0} J_{z} \cdot d S .
$$

The surface integral of $\nabla \times B$ is nonzero because the line integral contributions from the $\theta$ path add and those on the $r$ path cancel, where $r$ is at the centroid of the phase jump,

$$
\begin{aligned}
\oint \mathbf{B} \cdot d \mathbf{l} \approx & \int_{0}^{\Delta \theta} B_{\theta}(r+\Delta r) d \theta+\int_{r+\Delta r}^{r-\Delta r} B_{r} d r^{\prime} \\
& +\int_{\Delta \theta}^{0}\left(-B_{\theta}\right)(r-\Delta r) d \theta+\int_{r-\Delta r}^{r+\Delta r} B_{r} d r^{\prime} \\
\approx & 2 B_{\theta} r \Delta \theta .
\end{aligned}
$$

This $B_{\theta}$ discontinuity is necessary to support the axial wave currents $J_{z}$ associated with the KAW electrostatic $E_{z}$ and Landau damping, along with the consequent heating and current drive.

Figure 12 shows that the calculated $B_{\theta}(r \approx$ core, edge $)$ phases of the individual modes track each other for each toroidal mode number $(n=-1$ to -15$)$. Each core value (boxes) is approximately $\pi$ radians out of phase with the corresponding edge (diamonds) value. Calculations were for the discrete mode frequencies corresponding to maximum antenna loading, as indicated in Fig. 3(a).

Given the wave model and discrete spectrum we have presented, the edge phase of $B_{\theta}(r)$ appears to track the core phase of those discrete modes that exist. Therefore the edge measurement of a core dispersion relation is possible. The above signatures could conceivably be an accident of phase 
mixing by neighboring $n$ components. But as we saw in Figs. 9 and 10, spatial structures are similar for neighboring toroidal mode number $n$, so neighboring overlap would not change the story much.

\section{CONCLUSION}

To our knowledge the discrete mode structure of the Alfvénic ion-ion hybrid branch has never been measured before. As one increases $\omega$ or $|n|$ or $\left|k_{z}\right|$, the discrete mode series crosses the Alfvén continuum boundary, as we show in Figs. 2 and 3. As we see in Figs. 9 and 10 these wave modes have sufficient global character to exist in the core, but also retain remanent surface fields so that they are susceptible to pumping by an antenna at the plasma edge. In the final analysis, this may be why pumping at the transition between Alfvénic DAE and core GAE modes from the edge is a useful scenario for the Phaedrus-T tokamak.

The Phaedrus-T experiments presented here along with the heating and current drive results have been carried out in a significantly different regime than the TCA experiments ${ }^{13,16}$ that defined the genre of Alfvén heating experiments. Our wave frequency was near the cyclotron frequency $\omega / \omega_{c \mathrm{H}}(r=0) \approx 0.7 \lesssim 1$, and our toroidal mode numbers were large $|n| \gg 1$. The TCA frequency was very low $\omega / \omega_{c \mathrm{H}}(r=0) \approx 0.1 \ll 1$, with small toroidal $|n|=0,2,4$. This removes us from the MHD regime of TCA and others, and requires us to include finite $\omega / \omega_{c i}$ Hall term effects that are of the same order or larger than the toroidicity effects. For the Phaedrus-T frequency range and serendipitous small fraction of minority impurity ions, it may be that access to core GAE modes is more effective than was possible on the TCA tokamak. We argue that dispersion diagrams like Fig. 3 explain how this would facilitate access to the core using antennas on the edge, and may involve access to the ion Bernstein wave (IBW) at the mode conversion region.

It may also be why our heating and current drive results are more clear than the TCA ${ }^{13,16}$ experiments. For our tokamak experiment, the electron heating ${ }^{7-9}$ and current drive $^{10,11}$ effects were strongest at $\omega / \omega_{c \mathrm{H}}(r=0) \approx 0.7$. In Fig. 3(a), this would put our optimum regime at the upper frequency end of the DAE series, just as the DAE modes cross the continuum edge (at $n=-11,-12,-13$ ) into the region where GAE modes can exist at $n=-14,-15$. For other tokamaks this may also turn out to be a useful approach to pump RF power into the core, or to maintain Alfvén wave current drive off axis. ${ }^{10,11}$

We have shown how the more familiar Buchsbaum-Bers ${ }^{25}$ ion-ion $\left(k_{\perp} \rightarrow \infty\right)$ hybrid resonance is the parallel cutoff limit $\left(k_{\|} \rightarrow 0, S=0\right)$ of the general finite $k_{\|} \neq 0$ case, i.e. the Alfvénic ion-ion hybrid mode. Even though the Phaedrus- $\mathrm{T}$ experiment worked best with a $\mathrm{H}$ majority species, the explanations above do not require $\mathrm{H}$ majority plasmas. They only depend on two or more ion species with different gyrofrequencies and a minority fraction that sets $\omega_{i i}$ near the desired mode conversion location. For larger tokamaks such as Tokamak Fusion Test Reactor TFTR, ${ }^{32}$ ion-ion hybrid mode conversion scenarios have been exploited for radially localized electron heating and current drive.
In the search for electron current drive schemes, waves with low parallel phase velocity are ideal candidates because they interact with the large bulk electron distribution. The few choices consist of ion Bernstein waves (IBW), Alfvén waves (AW), kinetic AW (KAW), and the Alfvénic ion-ion hybrid waves discussed in this paper. In the usual Stix notation, the notion of a $k_{r} \rightarrow \infty$ perpendicular resonance at some $N_{\|}^{2}=S$ location holds equally well for the well-known Alfvén resonance and the ion-ion hybrid resonance. In these cases the IBW can appear between harmonics of the heavier species, so these waves are all related at the $N_{\|}^{2}=S$ resonance. Aside from the fact that the IBW occurs between gyroharmonics and the KAW is usually regarded as existing below the gyrofrequency $\omega<\omega_{c i}$, the physical manifestations of the two waves appear to be very similar. They are both highly damped, largely electrostatic, mostly radially propagating $k_{r} \gg k_{z}$ waves, with large $k_{r} \rho_{i} \approx 1$, where $\rho_{i}$ is the ion gyroradius. From the standpoint of the ISMENE code, we cannot decide whether to call the resonance and jump in the $B_{\theta}$ phase shown in Fig. 10 an IBW or KAW. The distinction between IBW and KAW may be more semantic than real.

At an aspect ratio of 3.7, the Phaedrus-T tokamak has significant toroidicity, yet a cylindrical model appears to sufficiently explain the data shown in this paper. The mode conversion waves occur in the core plasma, which has a large local aspect ratio, and may be why the perturbative treatment of toroidicity is adequate. A more complete discussion of when it is legitimate to invoke a cylinder approximation of the toroidal Alfvén geometry can be found in a thesis by Vukovic, ${ }^{33}$ and is based in part on earlier work by Hellsten and Tennfors, ${ }^{34}$ as well computer codes ISMENE $^{30}$ and LION. ${ }^{30}$ Vukovic ${ }^{33}$ also includes reflectometer data that shows the effects of varying minority concentration.

The dispersion relations $\omega\left(k_{z}\right)$ shown in Figs. 2 and 3 would seem to indicate slopes or group velocities $\partial \omega / \partial k_{z}$ greater than the speed of light, infinite or negative! This is actually a manifestation of the fact that when there is significant damping, neither the phase nor group velocity are useful measures of a wave envelope signal speed. ${ }^{31}$ Dissipation of the wave creates a gross distortion of wave packets, and the usual assumptions used to derive the propagation speed of the wave envelope and its energy are no longer valid. The data above in Figs. 3 and 4 show this anomaly to correlate with the measured strong damping.

In principle, there ought to be a connection between damping and propagation, as explained in our discussion of the fluctuation dissipation relations inherent in Fig. 5. Continuum shear Alfvén waves in a collisional plasma have also been shown to obey a similar paradigm. Amagishi and Tanaka ${ }^{35}$ demonstrated that a Kramers-Kronig relation connected the real and imaginary parts of a wave dielectric function.

In summary, we have shown that small fractions of minority ions can have a large effect on the Alfvén spectrum, as measured at the edge. We have used a low-power frequency sweeping technique and experimentally identified the ionion hybrid Alfvén wave that was probably observed by Borg and Cross. ${ }^{24}$ We have shown for the first time its discrete spectrum, which results from the presence of a minority ion 
species, and suggested a statistical fluctuation dissipation relation that relates fluctuations and wave damping.

\section{ACKNOWLEDGMENT}

This work was supported by the United States Department of Energy Grant No. DE-FG02-88ER53264.

${ }^{1}$ J. Tataronis and W. Grossman, Z. Phys. 261, 203 (1973).

${ }^{2}$ L. Chen and A. Hasegawa, Phys. Fluids 17, 1399 (1974).

${ }^{3}$ D. W. Ross, G. L. Chen, and S. W. Mahajan, Phys. Fluids 25, 652 (1982).

${ }^{4}$ J. M. Davila, Astron. Astrophys. 317, 514 (1987).

${ }^{5}$ A. Hasegawa and L. Chen, Phys. Fluids 19, 1924 (1976).

${ }^{6}$ R. L. Lysak and C. T. Dum, J. Geophys. Res. 88, 365 (1983).

${ }^{7}$ T. Intrator, P. Probert, M. Vukovic, A. Elfimov, R. D. Durst, S. Wukitch, M. Cekic, R. A. Breun, D. Brouchous, D. Diebold, M. Doczy, D. Edgell, R. Fonck, N. Hershkowitz, M. Kishinevsky, R. Majeski, C. Litwin, G. McKee, G. Winz, "Alfvén Ion Ion Hybrid Heating in the Phaedrus-T Tokamak," submitted to Phys Plasmas (1995).

${ }^{8}$ T. Intrator, P. Probert, M. Vukovic, S. Wukitch, A. Elfimov, R. D. Durst, R. A. Breun, D. Brouchous, D. Diebold, M. Doczy, R. Fonck, N. Hershkowitz, M. Kishinevsky, C. Litwin, P. Nonn, G. Winz, 11th Topical Conference on Radio-Frequency Power in Plasmas, AIP Conf. Proc. 355, edited by R. Prater and V. D. Chan, 17-19 May 1995, Palm Springs, CA (American Institute of Physics, Woodbury, NY, 1995), p. 221.

${ }^{9}$ R. Majeski, P. H. Probert, P. Moroz, T. Intrator, R. Breun, D. Brouchous, H. Y. Che, J. R. DeKock, D. Diebold, M. Doczy, R. Fonck, N. Hershkowitz, R. D. Johnson, M. Kishinevsky, G. McKee, J. Meyer, P. Nonn, S. P. Oliva, J. Pew, J. Sorenson, T. Tanaka, M. Vukovic, and G. Winz, Phys. Fluids B 5, 2506 (1993).

${ }^{10}$ T. Intrator, P. Probert, S. Wukitch, M. Vukovic, D. Brouchous, D. Diebold, R. Breun, M. Doczy, D. Edgell, A. Elfimov, N. Hershkowitz, M. Kishinevsky, C. Litwin, P. Moroz, P. Nonn, and G. Winz, Phys. Plasmas 2, 2263 (1995).

${ }^{11}$ S. Wukitch, M. Vukovic, R. Breun, D. Brouchous, D. Diebold, M. Doczy, A. Elfimov, D. Edgell, N. Hershkowitz, T. Intrator, M. Kishinevsky, C. Litwin, P. Moroz, and P. Probert, Phys. Rev. Lett. 74, 2240 (1995).

${ }^{12}$ R. D. Bengtson, J. F. Benesch, G. L. Chen, T. E. Evans, Y. M. Li, S. H. Lin, S. M. Mahajan, R. B. Michie, M. E. Oakes, D. W. Ross, P. Valanju, and C. M. Surko, Proceedings of the 3rd Joint International Symposium on Heating in Toroidal Plasmas, 22-26 March 1982, Grenoble [Association Euratom, Comissariat de l'Energie Atomique (CEA), Paris, France, 1982], Vol. 1, p. 151.

${ }^{13}$ G. A. Collins, F. Hoffman, B. Joye, R. Keller, A. Lietti, J. B. Lister, and A. Pochelon, Phys. Fluids 29, 2260 (1986); B. Joye, A. Lietti, J. B. Lister, J. M. Moret, and W. Simm, Phys. Rev. Lett. 56, 2481 (1986).

${ }^{14}$ A. B. Murphy, Nucl. Fusion 31, 465 (1991).

${ }^{15}$ P. Descamps, G. Van Wassenhove, R. Koch, A. M. Messiaen, P. E. Van- denplas, J. B. Lister, and Ph. Marmillod, Phys. Lett. A 143, 311 (1990).

${ }^{16}$ J. Vaclavik and K. Appert, Nucl. Fusion 31, 1945 (1991).

${ }^{17}$ S. M. Mahajan, Phys. Fluids 27, 2238 (1984).

${ }^{18}$ S. Rauf and J. Tataronis, Phys. Plasmas 2, 340 (1995).

${ }^{19}$ T. H. Stix, Waves in Plasmas, 2nd ed. (American Institute of Physics, New York, 1992).

${ }^{20}$ R. C. Cross, An Introduction to Alfvén Waves (Adam Hilger, Bristol, United Kingdom, 1988).

${ }^{21}$ See National Technical Information Service Document No. DE-82001702 (A. Hasegawa and C. Uberoi, The Alfvén Wave). Copies may be ordered from the National Technical Information Service, 5285 Fort Royal Road, Springfield, VA 22161.

${ }^{22}$ N. F. Cramer and C. M. Yung, Plasma Phys. Controlled Fusion 28, 1043 (1986).

${ }^{23}$ A. Elfimov, J. Tataronis, and N. Hershkowitz, Phys. Plasmas 1, 2637 (1994).

${ }^{24}$ G. G. Borg, R. C. Cross, Plasma Phys Controlled Fusion 28, 681 (1987).

${ }^{25}$ S. J. Buchsbaum, Phys. Fluids 3, 418 (1960).

${ }^{26}$ R. A. Breun, D. Brouchous, D. Diebold, R. Fonck, N. Hershkowitz, T. Intrator, Y. J. Kim, M. Kishinevsky, W. Li, R. Majeski, J. Pew, P. Probert, E. Y. Wang, Y. Wen, H. Che, M. Doczy, G. McKee, J. Sorenson, T. Tanaka, M. Vukovic, P. Bellan, and M. R. Brown, Fusion Technol. 19, 1327 (1991).

${ }^{27}$ T. Intrator, R. Majeski, P. Probert, and M. Vukovic, in Radio Frequency Power in Plasmas, Proceedings of the 9th Topical Conference, Charleston, 1991, AIP Conf. Proc. 244 (American Institute of Physics, New York, 1991), p. 319.

${ }^{28}$ N. Hershkowitz, R. Majeski, T. Tanaka, and T. Intrator, in Radio Frequency Power in Plasmas, Proceedings of the 8th Topical Conference, Irvine, 1989, AIP Conf. Proc. 190 (American Institute of Physics, New York, 1989), p. 270.

${ }^{29}$ T. Obiki, T. Mutoh, S. Adachi, A. Sasaki, A. Iiyoshi, and K. Uo, Phys. Rev. Lett. 39, 812 (1977).

${ }^{30}$ K. Appert, T. Hellsten, J. Vaclavik, and L. Villard, Comput. Phys. Commun. 40, 73 (1986); Th. Martin and J. Vaclavik, Helv. Phys. Acta 60, 471 (1987); L. Villard, K. Appert, R. Gruber, and J. Vaclavik, Comput. Phys. Rep. 4, 95 (1986).

${ }^{31}$ L. Brillouin, Wave Propagation and Group Velocity (Academic, San Diego, 1960)

${ }^{32}$ R. Majeski, C. K. Phillips, and R. Wilson, Phys. Rev. Lett. 73, 2204 (1994).

${ }^{33}$ M. Vukovic, "Toroidal effects on the Alfvén resonance in the Phaedrus-T tokamak," Ph.D. thesis, University of Wisconsin, Madison, WI, 1995.

${ }^{34}$ E. Tennfors, Plasma Phys. Controlled Fusion 28, 1483 (1986); T. Hellsten and E. Tennfors, Phys. Scr. 30, 341 (1984); T. Hellsten and E. Tennfors, in Proceedings of the 9th European Conference on Controlled Fusion and Plasma Physics (European Physical Society, Petit-Lancy, 1979).

${ }^{35}$ Y. Amagishi and M. Tanaka, Phys. Rev. Lett. 71, 360 (1993). 